\title{
Mascherine (in tempo di COVID-19 e comunicazione)
}

\author{
Leonardo Mari \\ Farmacia dell'Ospedale del Mugello, Azienda USL Toscana Centro, Firenze - Italia
}

Ultima maschera della finzione più bella fugge Arlecchino da questo Carnevale impaurito e triste, lo sguardo nascosto di chi vuol restare solo col proprio dolore.

Altre maschere coprono ora i volti senza coriandoli, diverse da sempre nello strascico della Quaresima di un Dio già malato a Natale.

Flebili voci infette alitano nell'aria i sospiri d'una libertà che manca girone d'un purgatorio in terra che non credevamo ma che meritiamo.

Urla da ogni dove la fame di tutto di sogni d'amore, d'un fresco e pulito domani che non verrà mai come un cammello non passa la cruna d'un ago.

E sempre più freddo stride tra i denti il falso sorriso che andrà tutto bene già manca da tempo la stretta di mano la parola che data non cambia nel tempo.

Piangiamo tutti, si piangono i morti spariti in fretta senza conforto nel breve deserto d'un sonno finto finito per sempre in un sacco chiuso.

Sono i giorni del falco che rotea nel cielo su canuti mercanti a contare denari, sono i giorni più adatti a capire chi siamo nel brusio delle voci che si leva lontano.

Received: May 27, 2020

Accepted: June 3, 2020

Published online: June 25, 2020

Indirizzo per la corrispondenza:

Leonardo Mari

SoS Farmacia

Ospedale del Mugello

Viale della Resistenza 60

50032 Borgo San Lorenzo, Firenze - Italia

leonardo.mari.56@gmail.com

\section{Commento a cura di Marco Lombardi \\ Editor in Chief, Giornale di Clinica Nefrologica e Dialisi}

La comunicazione, l'empatia e la capacità di stabilire una relazione non sono cose che i medici di solito imparano all'Università (1).

È spettacolare come in sette quartine di una poesia si possano raccontare così tante cose, "tutte cose".

Come scrive Sandro Spinsanti in "La medicina salvata dalla conversazione" (2) ... l'informazione circola, anche se la bocca rimane chiusa (ndr: cosa molto opportuna di questi tempi). Parlano gli occhi, il silenzio stesso parla, le pause sono molto eloquenti. Per non dire quanto può raccontare un sorriso o la sua assenza ...

Non si tratta di comunicazione numerica (dare un nome alle cose) si tratta piuttosto di una forma sublime di comunicazione analogica (rappresentabile in vario modo, in questo caso sotto forma di poesia), dove le quartine assumono, nel nostro pensiero, anche ciò che non è verbale e che arricchisce e colora "il verbo" (nella vita pre-COVID la posizione e la gestualità del corpo, le espressioni del volto, l'inflessione, il ritmo e la cadenza della voce e delle parole, ecc. ecc.), qui assolutamente la metafora, l'immaginifico, il bisogno dei versi.

Se il medico (per dire di un operatore della sanità) è spesso ancorato, per necessità, alla forma comunicativa numerica, quella più prettamente umana e che permette la trasmissione di informazioni precise (una classica anamnesi), ci sono situazioni e momenti particolari in cui prevale la forma comunicativa analogica, ovvero quella che abbiamo in comune con il mondo animale. Come scrive sempre Spinsanti, la morte e la nascita sono due situazioni in cui tipicamente prevale la comunicazione analogica, dove la "relazione" prevale sull'"informazione". Un'altra situazione in cui prevale certamente la comunicazione analogica è la malattia.

Prendersi cura di un malato: lo si può fare in tanti modi, riuscendo ad ascoltarlo, permettendogli di raccontarsi o con il raccontarsi dello stesso operatore, con dei gesti d'affetto, con un immagine e, perché no, con una poesia (scritta o letta).

Di questi tempi, purtroppo, non tutti I'hanno ancora compreso, il più illustre malato è il Nostro Pianeta e, di conseguenza, sono malati tutti quelli che lo abitano, imprescindibilmente da specie animali, vegetali, minerali e quant'altro. È un caso clinico in cui non si potrà prescindere 
da una comunicazione corretta e dalla centralità delle relazioni.

Chiudo questo cerchio scrivendo che, nella poesia, in questa poesia, io trovo relazioni e analogie tremende e vere (purtroppo) con la situazione attuale, pregressa e futura.

\section{Bibliografia}

1. Ofri D. Cosa dice il malato, cosa sente il medico. II Pensiero Scientifico Ed. 2018.

2. Spinsanti S. La medicina salvata dalla conversazione. II Pensiero Scientifico Ed. 2018. 